\title{
Preschool vision screening: negative predictive value for amblyopia
}

\author{
Douglas K Newman, Miranda M East
}

\begin{abstract}
Backgroundlaims-Single optotype tests of visual acuity are widely used for preschool vision screening in order to optimise cooperation with testing. These tests may, however, underestimate the visual acuity deficit in amblyopia because they lack visual crowding. This study assessed the resultant negative predictive value (NPV) for amblyopia.

Methods-Cohort study of 936 children in the Cambridge Health District selected by date of birth. The presence of amblyopia among children who had passed preschool vision screening was determined using Snellen line acuity as the reference test. Preschool vision screening was conducted at 3.5 years of age by community orthoptists. The screening assessment comprised Sheridan-Gardiner single optotype test of visual acuity (referral criterion $6 / 9$ or worse in either eye), cover test, ocular movements, $20^{\Lambda}$ prism test, and TNO ster-
\end{abstract} eotest.

Results-The overall NPV of preschool vision screening for amblyopia was $100 \%$ (95\% CI $99.4 \%$ to $100 \%)$. Most children with amblyopia were detected by the Sheridan-Gardiner single optotype test of visual acuity, but the other screening tests were necessary to prevent any false negatives. In isolation, the Sheridan-Gardiner single optotype test of visual acuity has a NPV for amblyopia of only $99.6 \%$ (95\% CI $98.7 \%$ to $99.9 \%)$.

Conclusion-Preschool vision screening using a single optotype test of visual acuity does achieve a high NPV for amblyopia, but only under certain conditions. These comprise a low threshold for referral (6/9 or worse in either eye) and the inclusion of a cover test and tests of binocular function in the screening assessment.

(Br F Ophthalmol 1999;83:676-679)

Ophthalmology,

Addenbrooke's

Hospital, Cambridge

CB2 2QQ

D K Newman

Community Child

Health, Lifespan

Healthcare, Ida

Darwin, Fulbourn,

Cambridge CB1 5EE

M M East

Correspondence to: D K Newman, Department of Ophthalmology, Clinic 3

(Box 41), Addenbrooke's

Hospital, Hills Road,

Cambridge CB2 2QQ.

Accepted for publication 18 December 1998 the Sheridan-Gardiner chart, are widely used for preschool vision screening in order to optimise cooperation with testing. ${ }^{3}$ These tests may, however, underestimate the visual acuity deficit in amblyopia because they lack visual crowding. ${ }^{4-6}$ In an attempt to prevent any children with amblyopia being missed by screening, a low threshold for referral (6/9 or worse in either eye) is generally adopted. Other components of the screening assessment, such as the cover test and tests of binocular function, may also help to reduce the risk of amblyopic children being missed by screening.

Children also undergo vision screening on school entry at 5-6 years of age. Visual acuity can be assessed at this age with linear optotype tests $^{27}$ which allow greater accuracy in the detection of amblyopia. Debate continues regarding the relative merits of preschool vision screening and vision screening at school entry for the detection and treatment of amblyopia since there are no controlled comparative studies. ${ }^{8}{ }^{9}$ The negative predictive value (NPV) for amblyopia is an important determinant of the relative efficacy of these screening options, but has received little attention to date. ${ }^{8}$ This study therefore assessed the NPV of preschool vision screening using a single optotype test of visual acuity.

\section{Methods}

A retrospective cohort study was conducted of children resident in the Cambridge Health District during 1995 with a date of birth in the 4 month period September to December 1986. These children were identified from the Community Child Health Service database which has a coverage of approximately $98 \%$. The population of the Cambridge Health District was 271000 . The preschool vision screening status of children in the cohort was determined by reviewing their community child health records.

Preschool vision screening is offered to all children in the Cambridge Health District at 3.5 years of age. The screening assessment (Table 1) is performed by an orthoptist in a community setting. The criteria for referral to the hospital eye service (HES) for further assessment are: (1) visual acuity $6 / 9$ or worse in either eye, (2) manifest strabismus, (3) decompensating heterophoria, (4) abnormality of ocular movements, (5) abnormal response to $20^{\Delta}$ base out prism test, (6) negative response

Table 1 Preschool vision screening assessment

Visual acuity test with Sheridan-Gardiner single optotype chart at 6 metres

Cover test at near and distance fixation

Ocular movements and convergence

$20^{\Delta}$ base out prism test

Stereoacuity test with TNO screening plate (1980 seconds of Stereo
arc) 
to TNO screening plate, or (7) any other ocular abnormality. In the presence of equivocal findings, children are recalled for another screening assessment by the community orthoptist.

The presence of amblyopia among children who had passed preschool vision screening was determined using Snellen line acuity as the reference test. Snellen line acuity was determined by reviewing the result of each child's school entry vision test at 5.5 years of age. If a Snellen line acuity of $6 / 6$ in each eye had not been documented, the child was recalled for another visual acuity assessment. Amblyopia was defined as a best corrected Snellen line acuity of $6 / 12$ or worse in either eye and/or an interocular difference of two Snellen lines or more.

The school entry vision test is conducted at 5.5 years of age in all maintained schools in the Cambridge Health District. Children in the cohort were therefore eligible for this test during the summer term of 1992 . Visual acuity is assessed by a school nurse using a full Snellen chart at 6 metres, with spectacle correction if worn. A Stycar single optotype test is employed for children unable to perform Snellen chart testing. Children found to have defective vision (and those unable to cooperate with Snellen line acuity testing) are generally retested by the school nurse on a subsequent occasion. Children with confirmed defective vision are referred, at the discretion of the school nurse, to either an optometrist or the HES.

The exact limits for the $95 \%$ confidence interval (CI) of the NPV for amblyopia were calculated using the binomial distribution. ${ }^{10}$

\section{Results}

There were 936 children in the selected cohort. The community child health records were reviewed for $95.9 \%$ (898/936) of these children. Of the remaining 38 children, 30 had left the Cambridge Health District and eight attended special schools for children with severe learning difficulties. The preschool vision screening status was available for $86.0 \%$ $(772 / 898)$ of the cohort (Table 2). Among these children, the attendance rate at screening was $77.3 \%(597 / 772)$ and the referral rate was

Table 2 Preschool vision screening status of children

\begin{tabular}{ll}
\hline Preschool vision screening status & No (\%) children \\
\hline Attended screening, passed & $542(60.4)$ \\
Attended screening, referred to HES & $55(6.1)$ \\
Defaulted screening & $157(17.5)$ \\
Already attending HES at age of screening & $18(2.0)$ \\
Unknown & $126(14.0)$
\end{tabular}

${ }^{\star} \mathrm{HES}=$ hospital eye service.

Table 3 Children with amblyopia who were not identified at preschool vision screening by the Sheridan-Gardiner single optotype test of visual acuity

\begin{tabular}{|c|c|c|c|}
\hline Child & $\begin{array}{l}\text { Reason for failing preschool vision } \\
\text { screening }\end{array}$ & HES findings & $\begin{array}{l}\text { Snellen line } \\
\text { acuity }^{\star}\end{array}$ \\
\hline 1 & $\begin{array}{l}\text { Decompensating esophoria }+ \\
\text { negative stereopsis }\end{array}$ & Anisometropic amblyopia & $6 / 96 / 5$ \\
\hline 2 & $\begin{array}{l}\text { Decompensating exophoria }+ \text { poor } \\
\text { motor fusion }\end{array}$ & $\begin{array}{l}\text { Unilateral posterior polar cataract } \\
\text { with amblyopia }\end{array}$ & $6 / 126 / 6$ \\
\hline
\end{tabular}

$\star$ Unaided Snellen line acuity recorded at HES before occlusion therapy was commenced. In both cases, the unaided visual acuity at preschool vision screening was $6 / 6$ in each eye with a SheridanGardiner single optotype test.
9.2\% (55/597). The preschool vision screening status of 126 children was not recorded in their community child health records. Some of these children would not have been invited to screening because they were not resident in the Cambridge Health District at 3.5 years of age, but the actual number could not be determined.

None of the 542 children who had previously passed preschool vision screening was found to be amblyopic. The NPV of preschool vision screening for amblyopia was therefore $100 \%$ (95\% CI $99.4 \%$ to $100 \%$ ). A Snellen line acuity of $6 / 6$ in each eye was achieved by 459 children at the school entry vision test, 75 children at a subsequent school vision test, and six children on retesting for this study. Six children required spectacle correction to achieve this level of acuity, but the nature of their refractive errors was not available. The best recorded visual acuity for the remaining two children was 6/9 6/9 Snellen and 6/6 6/6 Stycar, respectively. These two children were not available for retesting of their visual acuity because they had left the Cambridge Health District. No child had been referred to the HES with suspected amblyopia following school vision testing.

The HES findings for the 55 children who had failed preschool vision screening were: no abnormality (22), straight eyed amblyopia (13), refractive error (10), strabismus (6), congenital superior oblique palsy (two), strabismus + amblyopia (one), and unilateral congenital cataract + amblyopia (one). A total of 15 children with amblyopia were therefore detected by preschool vision screening. They had failed screening for the following reasons: reduced vision (10), reduced vision + decompensating heterophoria (two), reduced vision + decompensating heterophoria + poor motor fusion (one), decompensating heterophoria + negative stereopsis (one), and decompensating heterophoria + poor motor fusion (one). Two children with amblyopia had therefore not been identified at screening by the SheridanGardiner single optotype test of visual acuity (Table 3). Screening with a single optotype test of visual acuity in isolation has a NPV for amblyopia of only $99.6 \%$ (95\% CI $98.7 \%$ to 99.9\%).

Eighteen children were already attending the HES at the age of preschool vision screening. Their diagnoses were as follows: strabismus + amblyopia (10), strabismus (three), congenital cataract (two), persistent hyperplastic primary vitreous (one), optic nerve hypoplasia (one), and unknown (one).

\section{Discussion}

In this study, the NPV of preschool vision screening for amblyopia was $100 \%$ (95\% CI $99.4 \%$ to $100 \%$ ). The most effective screening test for the detection of amblyopia was visual acuity assessment, as reported previously. ${ }^{1}$ However, single optotype tests of visual acuity, even with a low threshold for referral (6/9 or worse in either eye), did not detect every child with amblyopia. Two of the 15 amblyopic children detected by preschool vision screening in 
this study were referred because of a decompensating heterophoria with reduced binocular function. Screening by visual acuity assessment alone would have resulted in a NPV for amblyopia of only $99.6 \%$ (95\% CI $98.7 \%$ to $99.9 \%$ ). While other screening tests contribute relatively little to the overall detection of amblyopia, ${ }^{1}$ they are necessary to minimise the risk of amblyopic children being missed by preschool vision screening.

The design of this study did not allow the NPV of preschool vision screening for its other main target conditions, strabismus and high refractive error, to be determined. Ocular alignment is not assessed in the school entry vision test. It is, however, unlikely that any children with strabismus were missed by preschool screening since ocular alignment was assessed by an orthoptist using the cover test. Refractive errors (as judged by wearing of spectacles) were present in six of the 542 children who had previously passed preschool vision screening. It is possible that these children had reduced vision due to uncorrected refractive errors at 3.5 years of age which was not detected at screening. Single optotype tests may certainly underestimate visual acuity deficits in the presence of uncorrected low refractive errors. ${ }^{4}$ However, these children may equally have developed refractive errors sufficient to reduce visual acuity after passing preschool vision screening.

There are some potential limitations in interpreting the results of this study. Firstly, Snellen line acuity was assessed by a retrospective analysis of testing performed by school nurses. This assessment was not performed under strictly standardised conditions, unlike preschool vision screening, with some consequent variability in Snellen chart design and testing conditions. Ascertainment of screening negatives should not, however, have been significantly impaired since visual acuity testing in schools tends to be performed under suboptimal conditions with consequent underestimation, rather than overestimation, of visual acuity. ${ }^{11}{ }^{12}$ Secondly, a Snellen line acuity was not available for one of the 542 children who had previously passed screening (his best recorded visual acuity was $6 / 6$ in each eye with a single optotype test). Amblyopia cannot be definitely excluded for this child which, if present, would reduce the NPV of preschool vision screening to $99.8 \%$ (95\% CI $99.0 \%$ to $100 \%)$. Thirdly, this study makes the assumption that no child was detected and treated for amblyopia in the 2 year interval between passing preschool vision screening and attending the school entry vision test. There is, however, no reason to doubt the validity of this assumption.

Few other studies have assessed the NPV of preschool vision screening using a single optotype test of visual acuity. ${ }^{13-15}$ In one Swedish study, ${ }^{13}$ visual acuity was assessed at 4 years of age with Marquez-Boström's hooks (a modification of the Landolt C optotype) using a referral criterion of $6 / 7.5$ or worse in either eye. The screening assessment also included the cover test and Wirt fly stereotest. Among an unselected cohort of 1530 children who had attended screening, only one child was found to have previously undetected amblyopia (secondary to anisometropia) on linear acuity testing at 7 years of age. Calculations based on the results reported in this study give a NPV for amblyopia of $99.9 \% \quad(95 \%$ CI $99.6 \%$ to $100 \%$ ), which is similar to our result. The findings of the other two studies are limited by inadequate ascertainment of screening false negatives ${ }^{14}$ and use of a single optotype test of visual acuity as the reference test for amblyopia. ${ }^{15}$

There have also been some reports of the NPV of preschool vision screening using a linear optotype test of visual acuity. ${ }^{16-18}$ One study assessed a screening programme in Cornwall which comprised a full orthoptic assessment that was otherwise almost identical to our study. ${ }^{16}$ It reported a $95 \%$ CI for the NPV for any target condition (amblyopia, refractive error or strabismus) in two separate study cohorts to be $99.12 \%$ to $99.25 \%$ and $99.46 \%$ to $99.54 \%$, respectively. These values are lower than our result, most probably due to the inclusion of refractive errors that developed after screening. Another study reported a NPV of $98.7 \%$ (95\% CI $95.4 \%$ to $99.9 \%$ ) for any target condition in a screening programme that comprised assessment of only visual acuity and stereoacuity. ${ }^{17}$ The findings of one further study are limited by inadequate ascertainment of screening false negatives. ${ }^{18}$

In addition to a high NPV, preschool vision screening should also have an acceptable positive predictive value (PPV). For the Cambridge Health District screening programme, a PPV of $79.9 \%$ (95\% CI $75.0 \%$ to $84.3 \%$ ) for the detection of any target condition (amblyopia, refractive error or strabismus) has previously been reported. ${ }^{19}$ Studies of other comparable, orthoptist based screening programmes have reported values ranging from $74 \%$ to 94\%. ${ }^{14}{ }^{16}{ }^{20}$ Apparently reduced vision is by far the most common reason for false positive referrals because of the low threshold for referral $\left(6 / 9\right.$ or worse in either eye).$^{19}$ This low threshold is necessary to prevent a significant proportion of children with amblyopia from being missed, ${ }^{1}$ but does not preclude a high PPV. An acceptable PPV is, however, only achieved when screening is performed by orthoptists, which reflects the expertise required to assess vision and ocular alignment accurately in young children. ${ }^{1421} 22$

A recent systematic review concluded that there is little evidence to support the proposed benefits of preschool vision screening. ${ }^{8}$ The current study provides evidence that the efficacy of amblyopia detection by preschool vision screening is equivalent to vision screening at school entry. Preschool vision screening with a single optotype test of visual acuity achieves a high NPV for amblyopia, provided tests of ocular alignment (cover test) and binocular function $\left(20^{\Delta}\right.$ prism test and stereoacuity tests) are also included. Another approach may be to use "crowded" optotype tests of visual acuity which are suitable for preschool children. ${ }^{323}$ While such tests have been shown 
to have greater sensitivity in detecting amblyopia, ${ }^{24}$ further validation studies are needed before they can be adopted for preschool vision screening. ${ }^{3}{ }^{7}$ Justification of preschool vision screening, however, still awaits the demonstration that earlier detection of amblyopia improves the visual outcome compared with vision screening at school entry.

Sources of funding: none.

Proprietary or financial interests: none.

1 Williamson TH, Andrews R, Dutton GN, et al. Assessment of an inner city visual screening programme for preschool children. Br F Ophthalmol 1995;79:1068-73.

2 Egan DF, Brown R. Vision testing of young children in the age range 18 months to $4^{1 / 2}$ years. Child Care Health Dev 1984;10:381-90.

3 Simons K. Visual acuity norms in young children. Surv Ophthalmol 1983;28:84-92.

4 Stuart JA, Burian HM. A study of separation difficulty. Its relationship to visual acuity in normal and amblyopic eyes. Am f Ophthalmol 1962;53:471-7.

5 Hilton AF, Stanley JC. Pitfalls in testing children's vision by the Sheridan Gardiner single optotype method. $\mathrm{Br} F \mathrm{Oph}$ thalmol 1972;56:135-9.

6 Youngson RM. Anomaly in visual acuity testing in children. Br F Ophthalmol 1975;59:168-70.

7 Salt AT, Sonksen PM, Wade A, et al. The maturation of linear acuity and compliance with the Sonksen-Silver acuity system in young children. Dev Med Child Neurol 1995;37 505-14.

8 Snowdon SK, Stewart-Brown SL. Preschool vision screening: results of a systematic review. York: NHS Centre for Reviews and Dissemination, 1997.

9 Rahi JS, Dezateux C. The future of preschool vision screening services in Britain. BMF 1997;315:1247-8.
10 Armitage P, Berry G. Statistical methods in medical research. 3rd ed. Oxford: Blackwell Sciences Ltd, 1994:118-25.

11 Ingram RM. Review of children referred from the school vision screening programme in Kettering during 1976-8. BMF 1989;298:935-6.

12 Yang YF, Cole MD. Visual acuity testing in schools: what needs to be done. BMF 1996;313:1053

13 Köhler L, Stigmar G. Visual disorders in 7-year-old children with and without previous vision screening. Acta Paediatr Scand 1978;67:373-7.

14 Jarvis SN, Tamhne RC, Thompson L, et al. Preschool vision screening. Arch Dis Child 1990;65:288-94.

15 Allen JW, Bose B. An audit of preschool vision screening. Arch Dis Child 1992;67:1292-3.

16 Wormald RPL. Preschool vision screening in Cornwall: performance indicators of community orthoptists. Arch Dis Child 1991;66:917-20.

17 De Becker I, MacPherson HJ, LaRoche GR, et al. Negative predictive value of a population-based preschool vision screening program. Ophthalmology 1992;99:998-1003.

18 Ingram RM, Holland WW, Walker C, et al. Screening for visual defects in preschoolchildren. Br F Ophthalmol 1986; 70:16-21.

19 Newman DK, Hitchcock A, McCarthy H, et al. Preschool vision screening: outcome of children referred to the hospital eye service. Br f Ophthalmol 1996;80:1077-82.

20 Köhler L, Stigmar G. Vision screening of four-year-old children. Acta Paediatr Scand 1973;62:17-27.

21 Edwards RS, Whitelaw AJ, Abbott AG. Orthoptists as pre-school screeners: a 2-year study. Br Orthopt f 1989;46: $14-9$.

22 Bolger PG, Stewart-Brown SL, Newcombe E, et al. Vision screening in preschool children: comparison of orthoptists screening in preschool children: comparison of orthoptists
and clinical medical officers as primary screeners. BMF 1991;303:1291-4.

23 McGraw PV, Winn B. Measurement of letter acuity in preschool children. Ophthalmic Physiol Opt 1995;15 Suppl 1:S11-S17.

24 Simmers AJ, Gray LS, Spowart K. Screening for amblyopia: a comparison of paediatric letter tests. $\mathrm{Br} \mathcal{F}$ Ophthalmol 1997;81:465-9. 